\title{
El proceso de institucionalización del Sistema Nacional de Educación Superior Ecuatoriano
}

\section{(The Institutionalization process of the Ecuadorian Higher Education National System)}

\author{
*Fabricio Guevara Viejo ${ }^{1}$, Leonidas Pacheco Olea ${ }^{1}$, Fernando Pacheco Olea ${ }^{1}$ \\ ${ }^{1}$ Universidad Estatal de Milagro \\ *jguevarav@unemi.edu.ec
}

\section{RESUMEN}

Desde el año 2008 el Sistema Nacional de Educación Superior (SNES) atraviesa un evidente proceso de cambio. Esta transformación se produce por la implementación de políticas del Estado que buscan calidad en la docencia, en la investigación y en su vinculación con la colectividad, sin embargo, luego de un extenso proceso de institucionalización cuyo desenlace a finales de la primera década del siglo XXI obligan al gobierno a tomar la drástica decisión de efectuar procesos de desinstitucionalización y de reinstitucionalización de forma simultánea que dan como resultado una nueva realidad institucional, cognitiva y social que encuentran resistencia en algunos sectores académicos con prácticas ya consolidadas producto del paso del tiempo y la inercia del sistema tradicional. Estudiaremos algunos de los procesos más relevantes de la institucionalización, desinstitucionalización y reinstitucionalización del Sistema Nacional de Educación Superior, y cómo estos configuran una realidad respecto a lo que esperan los actores políticos construida a partir de la resignificación de los actores involucrados.

Palabras clave: Teoría institucional, universidad, docencia, transformación educativa.

\begin{abstract}
Since 2008, the National System of Higher Education (SNES) has undergone a noticeable transitional process. This transition is the result of the implementation of some government policies which seek to achieve an enhancement in university teaching and research practice and their consistency with community involvement. Nevertheless, the outcomes obtained by the end of the 21st century, after an extensive process of institutionalization, forced the government to make the radical decision of applying certain processes of deinstitutionalization and re-institutionalization at the same time. Those processes resulted on a renewed institutional, cognitive, and social reality which finds some resistance in some academic areas as there are both practices and procedures that have been established over time by the inertia of the traditional system. We will explore and analyze some of the most relevant processes of institutionalization, deinstitutionalization, and re-institutionalization within the National System of Higher Education as well as how those processes create a reality which is built upon the expectations of stakeholders and the resignification that the actors involved in Ecuadorian universities give to this reality.
\end{abstract}

Keywords: Institutional theory, university, teaching, educational innovation. 


\section{INTRODUCCIÓN}

\section{La teoría institucional}

La teoría institucional surge como un cuestionamiento a la idea de que las organizaciones son sistemas cerrados exentos a la influencia del medio en que se desenvuelven y abre el camino para la sustentación de la teoría de sistemas abiertos. La teoría institucional es en la actualidad una de las principales orientaciones para concebir ideas acerca de las organizaciones ya que se dedica a estudiar la relación entre las organizaciones y su entorno mediante la evaluación de la igualdad en las organizaciones desde una perspectiva ambiental o del entorno (Greenwood, Oliver, Sahlin, \& Suddaby, 2009). Esta relación organización-ambiente plantea además otros dos puntos de vista importantes: de la organización propiamente dicha y de la ecología poblacional, que toma en consideración la diversidad en las organizaciones. Selznick (1957) en sus postulados sobre la teoría institucional menciona que una organización no solo se implanta dentro de un entorno, sino que interactúa de forma efectiva con el medio ambiente donde muestra símbolos y valores que se deben tomar en cuenta en la búsqueda de supervivencia y legitimidad.

\section{La institucionalización}

La institucionalización es el proceso a través del cual las organizaciones desarrollan un tipo de estructuras en las que se caracterizan, de forma distintiva, algunos "patrones de integración socialmente ordenados y estables" (Selznick, 1996: 21). La institucionalización también es definida como "el proceso mediante el cual las instituciones se construyen, con el fin de alcanzar un estado o propiedad deseada" (Lima, 2005: 25). También es considerada un proceso de adaptación a valores que se difunden por interacción social. Scott (1995) define la institucionalización como un proceso de adaptación a valores que se infunden por interacción social. (Selznick, 1957) menciona que la institucionalización constituye una idea neutral que se manifiesta con la aparición de patrones socialmente integrados, ordenados y estables que nacen de actividades técnicas usual- mente inestables, restringidas o con una débil organización.

Kostova y Dacin (2009) sostienen que la desinstitucionalización equivale al proceso por el cual las instituciones se debilitan y desaparecen en vista de que la legitimidad de una práctica institucional consolidada es interrumpida o deteriorada. Podemos deducir que es muy raro que las organizaciones sean totalmente consumadas, por el contrario, las instituciones que van extinguiendo no desaparecen completamente, su génesis, sus instancias permanecen latentes e influyen en la nueva institución.

Aun cuando se debiliten, las prácticas institucionales siempre conservan rezagos de su personalidad, de su estructura fundamental, mismo que actúa como límite histórico que sustenta el próximo paso, el proceso de reinstitucionalización (Kostova y Dacin, 2008).

El viejo institucionalismo definió a las instituciones como medios imperfectos pero perfectibles, capaces de lograr soluciones no siempre óptimas, pero encaminadas a la generación de beneficios para sus integrantes. (Vargas, 2008: 47). Concibe al ambiente como el lugar en que las organizaciones establecen lealtades nacidas de la cercanía o del trato cara a cara. Por su parte, "el nuevo institucionalismo considera a las organizaciones más que vinculadas a una comunidad local, vinculadas a un sector o área de la sociedad, similares a las que configuran industrias, profesiones o sociedades" (Meyer y Scott, 1983: 48).

El nuevo institucionalismo surge como una respuesta a ciertas incoherencias presentadas la práctica organizacional: políticos que defienden programas diseñados que nunca se ejecutan; gerentes compilando información que nunca se analiza, asesores expertos contratados que dan recomendaciones que nunca se siguen. Todo con la finalidad de exhibir legitimidad, probablemente esta situación se deba a que los instrumentos de análisis no están directamente vinculados a su entorno. El viejo institucionalismo atribuye al ambiente una presión considerable; el nuevo, lo considera sutil e indirecto. 


\section{El nuevo institucionalismo en el Sistema Nacio- nal de Educación Superior}

Para efectuar un estudio institucional, no es suficiente tomar en cuenta una disciplina, debemos observar desde diversos puntos de vista para lograr resultados más completos y precisos. Para este fin, el neo institucionalismo nos permite realizar una visión multidisciplinar. Guzmán (2004) sostiene que las estructuras normativa, reguladora y cognitiva de las universidades además de las estructuras culturales y rutinarias, a las que ella llama sus carriers, son muy similares entre las universidades, hecho que les confiere cierta homogeneidad.

Martínez (2000) menciona que esta homogeneidad universitaria se pone de manifiesto en la gestión administrativa de la organización. Una Facultad de 600 estudiantes, tiene la misma estructura que una de más de 50.000 alumnos. Insiste en que la cultura institucional de la universidad se desarrolla cotidianamente en base de acuerdos sobre los más importantes temas de discusión llegando a decisiones que muy poco alteran las viejas estructuras organizacionales, esta priorización del consenso frecuentemente hace improductiva la deliberación, inmoviliza los procesos decisorios y propende al crecimiento institucional por inercia.

Los isomorfismos vigentes en el Sistema Nacional de Educación Superior (SNES) son propicios para su institucionalización, pero condicionan su legitimidad por la exigente reglamentación, normativas y requisitos exigidos por la LOES, por ejemplo, los mecanismos de evaluación y acreditación. Las instituciones de educación superior (IES) estatales tienen exigencias adicionales como las auditorias por instituciones de control del Estado y la ejecución presupuestaria y financiera.

El espacio para innovación institucional en el Ecuador, algunas veces es restringido, en ocasiones percibido como una copia de sus pares occidentales, las propuestas e iniciativas de cambio son muchas veces vistas por el sistema como amenazas que ponen en peligro las estructuras institucionales. Martínez (2000) señala que los acuerdos a los que se llagan son solo circunstan- ciales, mismos que deben ser permanentemente revalidados. El equilibrio de fuerzas evita giros radicales en la orientación y en la toma de decisiones, pero en gran medida inhibe la innovación.

Cerrar una universidad es una ruptura institucional desde afuera, un proceso de desinstitucionalización para su transformación, esto no garantiza que la organización que tome su lugar esté exenta de los problemas seculares propios de universidad ecuatoriana tradicional. Scott (1995) sostiene que la universidad modela el comportamiento de sus integrantes en la práctica formal pero también en las actividades informales que estructuran el sentido de pertenencia en sus actividades que legitima la supervivencia. En este contexto, también los docentes, investigadores y estudiantes tienen un papel preponderante en la gestión, constituyen un instrumento estratégico para el logro de los objetivos institucionales.

Hunter, Bergesen y Kursweil (1984) mencionan que para reconocer el rol de los mecanismos institucionales en el cambio o en el mantenimiento de las prácticas institucionalizadas se necesita retornar el concepto de institucionalización entendido como un proceso a través del cual el cual los individuos que integran las instituciones han construido progresivamente una forma específica y tradicional de ser, que se pone de manifiesto en las estructuras fundamentales de las organizaciones.

\section{Procesos de reforma de la universidad ecuato- riana}

Durante la época de la Colonia española mediante decretos de las Cortes de Cádiz, se fundaron en Latinoamérica 32 universidades con la finalidad primordial de proveer instrucción a los novicios de las órdenes religiosas que cortejaban al conquistador español y satisfacer la creciente demanda que surge por el incremento del trabajo de evangelización y formación de los criollos e hijos de los blancos (Tunnerman, 1996). En 1596, durante la época en que la educación e instrucción era una ocupación exclusiva de la iglesia, se creó en Quito la primera universidad ecuatoriana bautizada con el nombre de San Fulgencio, luego los jesuitas en 1622 fundaron la Universidad San 
Gregorio cuya principal finalidad era la educación de los criollos.

El proceso de institucionalización de la universidad europea se desarrolló en función a dos modelos: el de París y el de Bolonia. Tunnermann (1996) afirma que la Universidad de París surgió de las escuelas catedralicias de Notre Dame, al servicio de las necesidades de adoctrinamiento en la Iglesia católica, su principal disciplina de estudio era la teología, con marcado privilegio de los pontífices en las decisiones doctrinales. La universidad de Bolonia, en cambio, surgió de la necesidad compartida de un grupo de estudiantes que buscaban profesores para poder ilustrarse por lo tanto fueron ellos los protagonistas en el gobierno y la administración de la institución. De allí surgen dos clásicos modelos universitarios: la Universitas Magistrorum (París) y la Universitas Scholarium (Bolonia).

En los primeros años de la etapa colonial, la presencia de religiosos instruidos en las aulas universitarias españolas, especialmente de Salamanca, manifestaba el propósito de incrementar el nivel de estudios y solicitar privilegios universitarios para conferir grados mayores. Posteriormente, ya en la Gran Colombia y ahora con los criollos, una nueva clase dominante en el poder, debido a las deudas de guerra y otros factores no fue posible sostener la educación pública.

La universidad, desde la perspectiva de $\mathrm{Na}$ poleón, debía tener un cuerpo docente que encaminara sus ideas morales y políticas. De forma contradictoria a los principios de igualdad, fraternidad y libertad que sustentaron su revolución, Napoleón exterminó los criterios que regían la universidad medieval: estudiantes y maestros en búsqueda de la verdad en libertad. Tornó la universidad en una institución de pobre desarrollo y la convirtió en una estructura utilitaria al servicio del Estado.

Las universidades de la recién independizada América Latina optaron por el modelo napoleónico. Se organizó en facultades, escuelas e institutos, pero obedeciendo más a un afán de control y menos a la creación de un sistema educativo que respondiera, con mente propia, a las realidades sociales y de desarrollo de los países recién libe- rados. La formación académica tuvo carácter general y se centró en las elites sociales, que eran las destinadas a gobernar los nuevos Estados.

Romero (2002) menciona que se pasó del modelo de Salamanca al modelo napoleónico de Francia, con preponderancia en estudios del derecho civil y las ciencias modernas desde enfoque científico positivista. En 1830, paralelo al nacimiento de la República del Ecuador, en el claustro universitario de la Universidad de Santo Tomás de Aquino, se inaugura una nueva etapa para la universidad ecuatoriana. Luego, en 1836, se promulgó la Ley Orgánica de Instrucción pública donde se busca la democratización del conocimiento y la autonomía universitaria.

Desde nuestra perspectiva, la universidad ecuatoriana sobrellevó cuatro etapas de reformas estructurales: la primera entre 1836 y 1901, lapso durante el cual se decretaron leyes con el fin de asumir el control de la educación superior para la República, alejarse de lo religioso y practicar el laicismo a través del control de nombramientos de docentes por parte del Estado. Entre 1920 y 1969 se da la lucha por la autonomía universitaria, a través de la promulgación de siete leyes de educación superior donde se evidenció que el tema central era establecer los límites de la autonomía y el gobierno de la IES ecuatorianas.

Una tercera etapa de reformas se produjo entre 1970 y 1990 donde se destaca la implementación de un sistema orientado al acceso libre que demanda la ampliación de los claustros docentes y mejoras en la infraestructura y un descenso en la calidad académica. Villavicencio, Sylva, Minteguiaga y Lima (2014) mencionan que en la universidad observaba una dicotomía respecto a su orientación hacia dos conceptos distintos de lo que es la educación superior (¿derecho o mercancía?), de lo que es el/la estudiante (¿ciudadano o cliente?) y la misma universidad (¿institución o empresa?).

Finalmente, la cuarta etapa de reformas se produce desde 2008, año de la promulgación de la actual Constitución, donde sobresale la demanda de eficiencia y eficacia para garantizar la pertinencia y la calidad de la educación superior 
hasta la actualidad.

\section{El sistema jurídico de la Educación Superior}

La Constitución de la República del Ecuador sitúa la educación como una actividad que responde al interés público, impide que sus objetivos sean manipulados u orientados para beneficiar a intereses de grupo. Garantiza la libertad de cátedra, la libertad, de enseñanza y el acceso a la educación en igualdad de condiciones. Los principios básicos sobre educación superior son expresados en 15 artículos de la Constitución.

La estructura del SNES refleja varios aspectos importantes: uno de estos es la proporción entre alumnos y profesores, que se inclina a favor de los primeros pero tienen una presencia mínima frente al número de gestores (rectores de las IES) quienes predominan en el escenario del asesoramiento frente al resto de los actores de la comunidad universitaria.

Otro aspecto importante es la estructura de asesoramiento, esta gravita en una proporción de 5 a 1 a favor de los gestores y en detrimento de los actores principales: docentes y estudiantes. Quienes se dedican a asesorar sobre el desarrollo del SNES son principalmente los rectores, tanto los de universidades (que están presentes en su totalidad en la Asamblea Universitaria) como los de los institutos (un total de 8 de los 195 institutos que hay en el país).

Del análisis de la organización de los órganos rectores y planificadores (CES y CEAACES), es significativo que se utilice el término académico diferenciándolo del término profesor (tal y como aparece en el órgano asesor). Por ello podemos inferir que están incluyendo en la categoría académicos a los profesores y gestores (rectores $\mathrm{u}$ otros).

Por otra parte, a pesar de que los académicos están representados en mayor número, en el CES es muy relevante el peso del ejecutivo, que alcanza una proporción de 2 a 3. Esto es, 4 representantes del ejecutivo frente a 6 académicos (profesores y/o gestores). Sin embargo, en el CEAACES, la proporción entre el ejecutivo y los académicos es de 1 a 1 ( 3 representantes del ejecutivo y 3 académicos). Podemos concluir que tanto el órga- no que asesora como los órganos que planifican la vida y el desarrollo del SNES recaen fundamentalmente en el poder político y en la alta gestión de las universidades.

La SENESCYT, el CEAACES, el CES, la SENPLADES, la Asamblea Universitaria, los Comités Regionales y las Redes Universitarias constituyen una forma típica de administración pública consultiva, en que los agentes del Estado conservan para sí la prerrogativa de tomar decisiones individuales que aporten a la construcción de un sistema de educación superior adecuado a los requerimientos del país.

El 12 de octubre de 2010, la Asamblea Nacional pone en vigencia la nueva Ley Orgánica de Educación Superior (LOES) diseñando un esquema institucional sólidamente estructurado que se rige por los principios de: Autonomía responsable; Cogobierno; Igualdad de oportunidades; Calidad; Pertinencia; Integridad; Autodeterminación. Con una estructura jerárquica de instituciones que rigen el Sistema Nacional de Educación Superior (SNES). Además de un rígido proceso de intervención, suspensión y extinción a las universidades y escuelas politécnicas. Además, un procedimiento para las sanciones.

Con la posibilidad de crear otros instrumentos normativos, administrativos, ejecutivos e independientes, de acuerdo a la necesidad del mismo sistema, que son responsabilidad del CES. Esta atribución está siendo desarrollada a través de sus consejeros que, luego de un proceso de sociabilización, que suele incluir el debate a nivel nacional de las diversas temáticas de interés, emiten el conjunto requerido de normativas. Entre los años 2010 y 2016, se aprobaron la totalidad de estos instrumentos jurídicos.

\section{EL Reglamento de Carrera y Escalafón del Do- cente e Investigador (RCEDI)}

Sylva (2014) menciona que el RCEDI regula la selección, ingreso, dedicación, estabilidad, escalas remunerativas, capacitación, perfeccionamiento, evaluación, promoción, estímulos, cesación y jubilación del personal académico de las universidades y escuelas politécnicas públicas y particulares en Ecuador, que representaban en 
el año 2012 una población de 33.720 personas.

Esta normativa regula la actividad de profesoras y profesores, investigadores e investigadoras, e incide en el cambio de paradigma, en el modo de entender la universidad y el conocimiento; se sustenta en el esfuerzo colectivo por situar a la universidad ecuatoriana al nivel de calidad de sus pares en el exterior; poniéndola, a la vez, al servicio de las necesidades sociales y productivas del país, mediante una revalorización de la figura del docente y una apuesta por la transformación de la figura del investigador.

De forma general, el RCEDI se instituye como una declaración de ruptura con el pasado de la universidad ecuatoriana. Villavicencio et al (2014) indica que los principios economicistas por los que se regía la universidad, especialmente a partir de los años noventa, dejan su lugar a los principios democratizadores. Si antes proliferaron universidades particulares que respondían a la explotación de un nicho de mercado concreto y sin atención especial a la calidad de la enseñanza; ahora se prioriza la democratización institucional; es decir, la revalorización del docente académico y del principio meritocrático.

La aprobación del RCEDI significa recuperar el sentido de universidad del pasado para proyectarla al futuro. Este instrumento normativo se convierte en la política pública que estaba ausente "orientada a establecer parámetros universales de desenvolvimiento de las IES en torno a sus principios, sus paradigmas educativos y organizativos y sus criterios de calidad" (Villavicencio et al, 2014:11).

Para fundamentar la democratización, el RCEDI sitúa al docente como el eje central para la transformación de la universidad ecuatoriana y en consecuencia del desarrollo del país. Uno de los objetivos principales del RCEDI es dar valor a la actividad profesional que se desarrolla en la universidad. Es también lograr que la universidad inserte el talento humano en su columna vertebral al situar a su personal docente en el centro de su configuración, definiendo sus actividades, su remuneración, los procesos de promoción interna y estimulación, e incidiendo en sus funciones investigadoras. Así, la actividad docente deja de ser precaria y pasa a convertirse en una actividad exclusiva y reconocida como motor central de la transformación del país.

El RCEDI, desde el punto de vista de la formación, estableció una ruptura con el sistema anterior marcado por un procedimiento formativo caracterizado por la desactualización bibliográfica y precarización laboral del docente. A través de su aplicación se pretende lograr profesoras/ es que estén actualizados/as, que generen conocimiento y que desarrollen tecnología e investigación; una transformación del profesorado que conlleve la transformación del alumnado.

Desde la perspectiva de los méritos exigidos, el RCEDI, pretende lograr el desarrollo del país a través de la exigencia de acciones y transformaciones urgentes que demandan grandes esfuerzos personales y organizacionales, particularmente a los docentes quienes, en algunos casos, creen que poner la universidad ecuatoriana al nivel de las universidades que, en varios contextos, nos están sirviendo de modelo -como las americanas o las europeas- requiere por lo menos el mismo tiempo que han tenido dichos países para desarrollar sus IES como centros de conocimiento y desarrollo.

La universidad ecuatoriana con el paso del tiempo se transformó en una organización de bajo nivel marcada por la masificación especialmente desde los años 70 con la numerosa llegada de estudiantes sin una previa planificación, en un ambiente de escasa pertinencia dentro de un marco legal acomodado a los requerimientos mercantilistas de grupos de poder económicos que convirtieron la educación superior en un lucrativo negocio.

El Gobierno inició entonces un proceso de desinstitucionalización de esas estructuras organizacionales caducas e ineficientes con la expedición del Mandato constituyente \#14 orientado a recuperar el rol director, regulador y supervisor del Estado sobre las IES, muy venido a menos durante décadas de gobiernos que renunciaron a su regulación dejándolo solamente supeditado a las fuerzas del mercado, sin embargo, la Asamblea Constituyente de 2008 declaró a la educación superior un bien público prioritario para el Estado, 
delegando la planificación, coordinación, regulación y evaluación de la universidad en tres organismos: CES, CEAACES y SENESCYT.

En 2008 se inició un inició un proceso de transformación que convirtiera a la educación superior en motor del cambio de la matriz productiva contemplada en el Plan del Buen Vivir. Pasamos de una universidad débil y deslegitimada a una universidad que lidera la compleja empresa de alcanzar el Buen Vivir.

La universidad tradicional entonces relegada y deslegitimada se transformó, no totalmente como el gobierno lo había previsto puesto que aún quedan remanentes del pasado que conviven con la nueva institucionalidad, lo que genera un proceso de reinstitucionalización.

Con la inclusión de la autonomía responsable en la normativa constitucional se legitima la integralidad en el aspecto académico, financiero, orgánico y administrativo, estos principios se ejercen poniendo en práctica la transparencia y fiscalización del patrimonio estatal, además de una permanente rendición de cuentas. En el ámbito académico es prioritario el principio de calidad, el cual se pone en práctica con el fortalecimiento del sistema de evaluación y acreditación institucional mediante el control de entes conformados por integrantes que no están relacionados con las IES que serán sometidas a control.

El escalafón docente de la universidad tradicional estaba marcado fundamentalmente por la antigüedad (años de trabajo). En el escenario actual se prioriza la formación a través de exigencias propias de la carrera académica. Esta innovación exige un cambio sustancial en la mentalidad de los docentes en relación directa con la tradición institucional de la que proceden.

La evidente transformación ocurrida en el Sistema Nacional de Educación Superior genera disyuntivas y origina conflictos en las relaciones de poder producto del impacto producido por la convivencia de los tres procesos de cambio organizacional: de institucionalización, de desinstitucionalización y de reinstitucionalización en un lapso de tiempo tan corto. En este contexto se crea una nueva realidad institucional generada a través de las resignificación del discurso y el esfuerzo personal de los integrantes de la organización.

Las precarias condiciones laborales de los docentes mejoraron sustancialmente desde octubre de 2012 luego de la promulgación del RCEDI, documento donde se regulan las actividades docentes y de investigación y vinculación con la colectividad. También se establecen niveles y escalas salariales estandarizadas en función, sobre todo, de los méritos académicos y el esfuerzo personal de los docentes universitarios y no solamente la visión tradicional de incrementos salariales en función de los años de trabajo.

El cambio institucional del SNES desecha sutiles transformaciones con el fin de lograr nuevas estructuras y cultura institucional utilizando sus propias prácticas y mecanismos institucionalizantes obligatorios que a la vez generan compromiso para la organización, para sus integrantes y para la comunidad. La actitud positiva en la construcción de un proyecto educativo, de investigación, vinculación y gestión con nuevos conceptos y el nuevo lenguaje que reconoce las complejidades y particularidades del sistemas, son el camino más propicio para realizar un ejercicio de resignificación que permita, en corto plazo, comprender las posibles ambigüedades del discurso educativo.

La transformación del SNES tiene como sustrato ideológico lo que se conoce como Socialismo del siglo XXI. El proyecto político de la llamada Revolución Ciudadana ha impulsado un cambio radical en este sistema con el diseño y puesta en marcha desde 2008 de una nueva normatividad sustentada en la Constitución a través de la LOES, el RCEDI, los diversos reglamentos y normativas que establecen una fuerte regulación en todos los procesos académicos, con el respeto a la autonomía universitaria supervisada y evaluada por los organismos rectores de sistema. De esta forma se logró romper la desregulación y la hegemonía del mercado sobre la educación superior derivada del modelo neoliberal. Como conclusión, el cambio estructural del SES ecuatoriano se ha desarrollado bajo tres parámetros clave:

1. Considerar la educación como un bien público de prioridad nacional.

2. Educación universitaria gratuita y univer- 
sal hasta el tercer nivel.

3. La educación superior como motor del desarrollo del país.

\section{REFERENCIAS BIBLIOGRÁFICAS}

Greenwood, R., Oliver, C., Sahlin, K. \& Suddaby, R. (2008). Introduction. En R. Greenwood, C. Oliver, R. Suddaby, \& K. Sahlin, (Eds.). The Sage Handbook of Organizational Institutionalism. London UK: Sage Publication.

Guzman, M. (2004). El nuevo institucionalismo como enfoque teórico para analizar el sistema universitario argentino. Buenos Aires: PIDE-UNTREF / UNLA.

Kostova, T., Roth, K. \& Dacin, M. T. (2008). Institutional theory in the study of multinational corporations: A critique and new directions. Academy of management review, 33(4), 994-1006.

Kostova, T., Roth, K. \& Dacin, M. T. (2009). Theorizing on MNCs: A promise for institutional theory. Academy of Management Review, 34(1), 171-173.

Martínez, N. (2000). La evaluación de la gestión universitaria. Buenos Aires: Documentos de la CONEA.

Scott, R. (1991). Institutions and Organizations. Oaks: Sage.

Searle, J. (1995). La búsqueda del significado. Lecturas de filosofía del lenguaje. Madrid: Tecnos.

Selznick, P. (1957). Leadership Leadership in Administration: A Sociological Interpretation. New York [ua].

Selznick, P. (1996). Institutionalism old and new. Administrative science quarterly, 270-277.

Sylva, E. (2014). Ecuador: Género y calidad en la docencia universitaria (2008-2012). En VV. AA. Calidad de la educación superior y género, 391426. Quito: Flacso.

Tünnermann, C. (1996). Breve historia del desarrollo de la universidad en América Latina. Caracas: CRESALC.

Vargas, J. (2008). Perspectivas del institucionalismo y neoinstitucionalismo. Ciencia Administrativa, (1), 47-58.

Villavicencio, A., Sylva, E., Minteguiaga, A,.
Lima, M. (2014) Evaluación de la Calidad de la Universidad Ecuatoriana. La experiencia del Mandato 14. CEAACES. Quito. Pp. 253.

\section{Cumbres}

